\title{
Atom TRANSFER RADical POLymeRIzation by CONTINUOUS FEEDING OF ACTIVATORS
}

LOUISIANTA
Yu Wang*, Alec Clay and Mary Nguyen Department of Chemistry, University of Louisiana at Lafayette, Lafayette, LA 70504, USA

\section{INTRODUCTION}

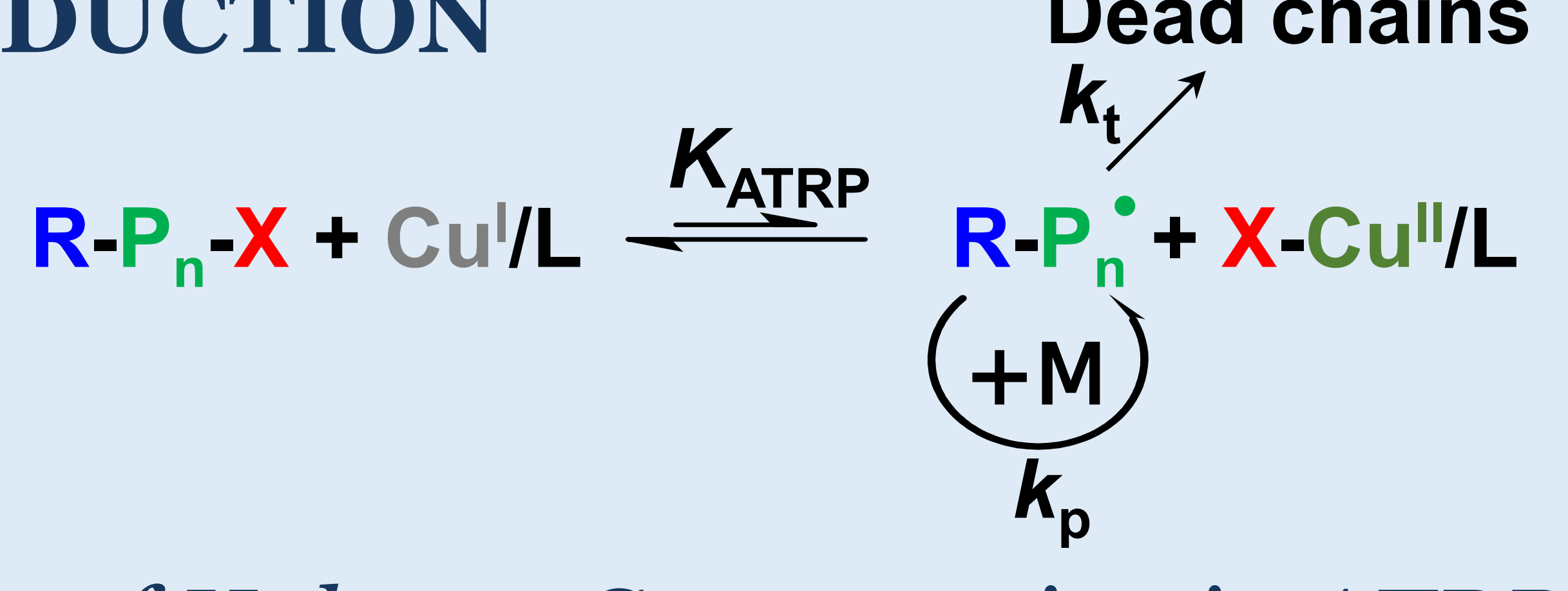

\section{Principle of Halogen Conservation in ATRP}

One critical issue in ATRP, as well as in any other RDRP, is that the polymer propagation is always accompanied by radical termination. Because the total amount of halogen in a reaction system should be constant, the chain end halogen group loss must result in the migration of the halogen atom and the oxidation of some other species in the reaction system. In normal ATRP, the result is some of the $\mathrm{Cu}(\mathrm{I}) \mathrm{X}$ would be permanently oxidized to $\mathrm{Cu}(\mathrm{II}) \mathrm{X}_{2}$.

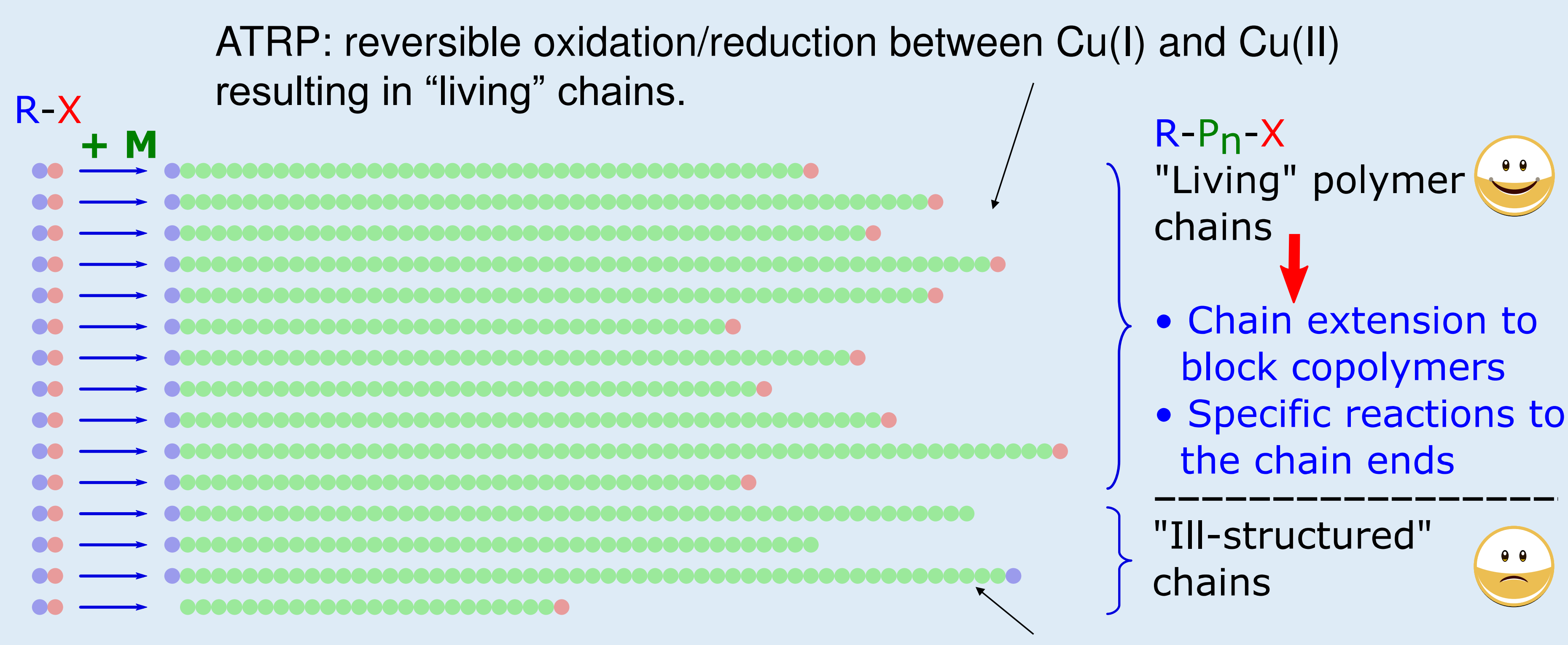

End-group loss = Irreversible oxidation from $\mathrm{Cu}(\mathrm{I})$ to $\mathrm{Cu}(\mathrm{II})$.

Limit the End-Group Loss

If the amount of $\mathrm{Cu}(\mathrm{I})$ used in ATRP is less than the amount of the initiator (R-X), the maximum potential mole percent of end-group loss, $T_{\text {mol\% }}$, would be limited to [Cu(I)]/[R-X]. This provides a way to conduct ATRP with predictable end-group fidelity. To use the $\mathrm{Cu}(\mathrm{I})$ catalyst most efficiently, continuous feeding of the activators was introduced.

$$
\mathrm{Cu}(\mathrm{I}) / \text { Ligand }
$$

\section{Limited mole percent of end-group loss}

$$
T_{\text {mol\% }} \leq \frac{[\mathrm{Cu}(\mathrm{I})]}{[\mathrm{R}-\mathrm{X}]}
$$

(3)

\section{RESULTS}

Polymerization of Methyl Acrylate (MA)

$\boldsymbol{D P}_{\mathrm{T}}$ time conv $\boldsymbol{M}_{\mathrm{n}, \mathrm{th}} \quad \boldsymbol{M}_{\mathrm{n}, \mathrm{GPC}} \boldsymbol{M}_{\mathrm{w}} / \boldsymbol{M}_{\mathrm{n}}$ $\begin{array}{lllllll}50 & 2 \mathrm{~h} & 86 \% & 3900 & 3700 & 1.15\end{array}$ $\begin{array}{llllll}100 & 2 \mathrm{~h} & 92 \% & 8100 & 8200 & 1.13\end{array}$ $100 \quad 4 \mathrm{~h} \quad 87 \% \quad 7700 \quad 7900 \quad 1.10$ $\begin{array}{llllll}100 & 8 \mathrm{~h} & 94 \% & 8200 & 9600 & 1.10 \\ 200 & 2 \mathrm{~h} & 84 \% & 14600 & 15100 & 1.10\end{array}$ $\begin{array}{llllll}50^{\mathrm{c}} & 2 \mathrm{~h} & 73 \% & 6500 & 7300 & 1.09\end{array}$

\begin{tabular}{|l|l|}
$\boldsymbol{T}_{\mathbf{m o l} \%, \mathbf{H C}}$ & $\boldsymbol{T}_{\mathbf{m o l} \%, \mathbf{N M R}} \boldsymbol{b}$ \\
\hline $\mathbf{2} \%$ & $3 \%$ \\
\hline $4 \%$ & $5 \%$ \\
\hline $4 \%$ & $6 \%$ \\
\hline $4 \%$ & $4 \%$ \\
\hline $8 \%$ & $6 \%$ \\
\hline $6 \%$ & $\sim 0 \%$ \\
\hline
\end{tabular}

CFA ATRP of MA. $[\mathrm{MA}]_{\mathrm{O}}:\left[\mathrm{CuBr}_{2}\right]_{\mathrm{o}}:\left[\mathrm{Me}_{6} \mathrm{TREN}_{\mathrm{O}}=100: 0: 005:\right.$ o:01 with 10ommol MA and 2, 1 and $0.5 \mathrm{mmol} \mathrm{MBrP,} \mathrm{with} 5 \mathrm{~mL}$ DMF in a Schlenk flask, stirring at $60^{\circ} \mathrm{C} .2 \mathrm{~mL} \mathrm{CuBr} / \mathrm{Me}_{6}$ TREN 0.02 $\mathrm{mol} / \mathrm{L}$ in $\mathrm{MeCN}$ was placed in a Hamilton airtight syringe and added at a constant addition rate in $2-8$ hours by using a syringe pump. $\boldsymbol{a}$ $T_{\mathrm{mol} \%}$ calculated by halogen conservation. $\boldsymbol{b} T_{\mathrm{mol} \%}$ determined by ${ }^{1} \mathrm{HNMR}$. $\boldsymbol{c}$ Chain extension from $\mathrm{PMA}_{43}-\mathrm{Br}$.

Polymerization of Methyl Methacrylate (MMA)

$$
\begin{aligned}
& \begin{array}{llllllll}
D P_{\mathrm{T}} & \text { time } & \text { conv } & M_{\mathrm{n}, \text { th }} & M_{\mathrm{n}, \mathrm{GPC}} & \boldsymbol{M}_{\mathrm{w}} / \boldsymbol{M}_{\mathrm{n}} & \boldsymbol{T}_{\mathrm{mol} \%, \mathrm{HC}}{ }^{\boldsymbol{a}}
\end{array} \\
& \begin{array}{llllllll}
50 & 23 \mathrm{~h} & 59 \% & 3152 & 4200 & 1.27 & <4 \% \\
100 & 22 \mathrm{~h} & 74 \% & 7608 & 8000 & 1.22 & <8 \%
\end{array} \\
& \begin{array}{llllllll}
100 & 22 \mathrm{~h} & 74 \% & 7608 & 8000 & 1.22 & <8 \% \\
200 & 23 \mathrm{~h} & 88 \% & 17890 & 19700 & 1.16 & <16 \%
\end{array} \\
& \begin{array}{lllllll}
200 & 23 \mathrm{~h} & 88 \% & 17890 & 19700 & 1.16 & <16 \% \\
400 & 23 \mathrm{~h} & 90 \% & 36062 & 42700 & 1.15 & <32 \%
\end{array}
\end{aligned}
$$

\begin{tabular}{|c|c|c|c|c|c|c|}
\hline$D P_{T}$ & time & conv & $M_{\mathrm{n}, \mathrm{th}}$ & $M_{\mathrm{n}, \mathrm{GPC}}$ & $M_{\mathrm{w}} / M_{\mathrm{n}}$ & $T_{\mathrm{mol} \%, \mathrm{HC}}{ }^{a}$ \\
\hline 50 & $8 \mathrm{~h}$ & $37 \%$ & 2047 & 1400 & 1.45 & - \\
\hline 50 & $22.5 \mathrm{~h}$ & $74 \%$ & 3987 & 3600 & 1.20 & $<4 \%$ \\
\hline 100 & $8 \mathrm{~h}$ & $34 \%$ & 3727 & 3300 & 1.30 & - \\
\hline 100 & $22 \mathrm{~h}$ & $67 \%$ & 7107 & 7400 & 1.16 & $<8 \%$ \\
\hline 200 & $8 \mathrm{~h}$ & $37 \%$ & 7778 & 6800 & 1.20 & - \\
\hline 200 & $23 \mathrm{~h}$ & $73 \%$ & 15277 & 17000 & 1.16 & $<16 \%$ \\
\hline
\end{tabular}

CFA ATRP of MMA. [MMA $]_{\mathrm{O}}:\left[\mathrm{CuCl}_{2}\right]_{0}:[\mathrm{TPMA}]_{\mathrm{O}}=100: 0: 0025:$ $0: 003$ at $80^{\circ} \mathrm{C}$ with 100mmol MMA and 2, 1, 0.5 and $0.25 \mathrm{mmol}$ EClPA, in $5 \mathrm{~mL}$ of DMF; $0: 04 \mathrm{mmol} \mathrm{CuCl} / \mathrm{TPMA}$ in $2 \mathrm{~mL}$ of $\mathrm{MeCN}$ was added in 8 h. $\boldsymbol{a} T_{\text {mol\% }}$ calculated by halogen conservation.

Polymerization of Styrene (St)

CFA ATRP of St. $[\mathrm{St}]_{\mathrm{O}}:\left[\mathrm{CuCl}_{2}\right]_{\mathrm{o}}:[\mathrm{TPMA}]_{\mathrm{o}}=100: 0: 0025: 0: 003$ at $110^{\circ} \mathrm{C}$ with $100 \mathrm{mmol} \mathrm{St}$ and 2,1 , and $0.5 \mathrm{mmol} \mathrm{PECl}$, in $5 \mathrm{~mL}$ of DMF; 0:04 mmol CuCl/TPMA in 2mL of MeCN was added in $8 \mathrm{~h} . \boldsymbol{a}$ $T_{\text {mol\% }}$ calculated by halogen conservation.

Abbreviations

MBrP: methyl 2-bromopropionate; $\mathrm{Me}_{6}$ TREN: tris[2(dimethylamino)ethyl]amine; EClPA: ethyl 2-chlorophenylacetate; TPMA: tris(2-pyridylmethyl)amine; PECl: (1-chloroethyl)benzene; $D P_{\mathrm{T}}$ : targeted degree of polymerization; conv: monomer conversion.

\section{Chain Extension}

Two similar macroinitiators, i.e., PMMA- $1 M_{\mathrm{n}}=19700, M_{\mathrm{w}} / M_{\mathrm{n}}=$ $1.16, T_{\mathrm{mol} \%}<16 \%$; and PMMA-2 $M_{\mathrm{n}}=23500, M_{\mathrm{w}} / M_{\mathrm{n}}=1: 16$, $T_{\text {mol\% }}<32 \%$, were synthesized by CFA ATRP. Chain extension was carried out with the ratio of reagents [MMA] : [PMMA-Cl] :

$\left[\mathrm{CuCl}_{2}\right]:[\mathrm{TPMA}]=400: 1: 0: 0025: 0: 003$ at $80^{\circ} \mathrm{C}$ with 100 mmol MMA in 5mL of DMF; 0:04 mmol CuCl/TPMA in 1mL $\mathrm{MeCN}$ was added in $4 \mathrm{~h}$. The resulting polymer PMMA-b-PMMA1: $M_{\mathrm{n}}=53400, M_{\mathrm{w}} / M_{\mathrm{n}}=1: 30, T_{\mathrm{mol} \%}<32 \%$; PMMA-b-PMMA-2: $M_{\mathrm{n}}=61000, M_{\mathrm{w}} / M_{\mathrm{n}}=1: 41, T_{\mathrm{mol} \%}<48 \%$. Apparently PMMA-1 resulted in less dead chains.
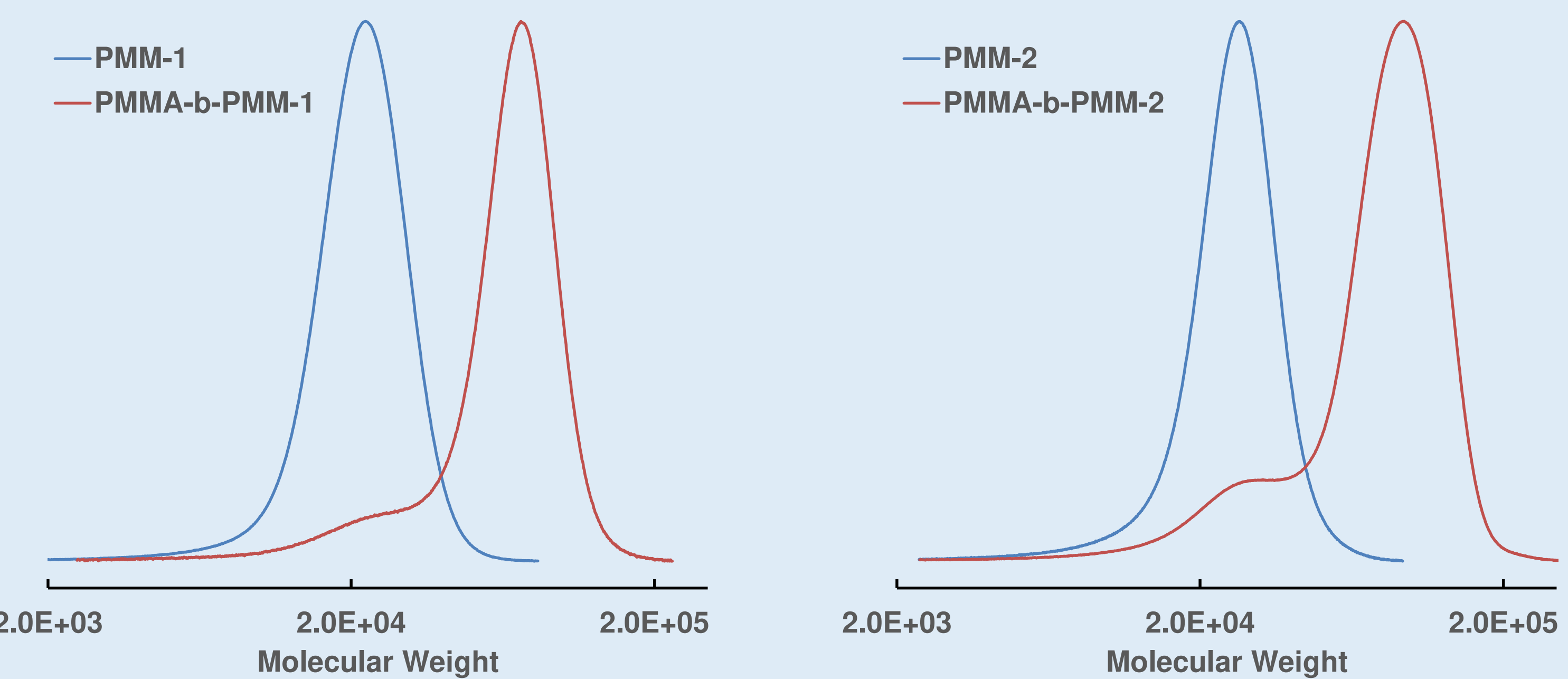

CONCLUSIONS

- ATRP by continuous feeding of activators provided a way to synthesize polymers with precisely-controlled end-group fidelity.

- CFA ATRP proved a robust technique for the polymerization of MA, MMA and St.

- This technique would be even more valuable when the endgroup analysis by ${ }^{1} \mathrm{HNMR}$ and MALDI-TOF is very difficult, e.g., for polymers with very high molecular weight, block polymers, polymers with labile end groups, polymers with complex architectures.

\section{REFERENCE:}

1. Y Wang*. ATRP of Methyl Acrylate by Continuous Feeding of Activators Giving Polymers with Predictable End-Group Fidelity. Polymers 2019, 11, 1238.

2. Y Wang*, A Clay, M Nguyen. ATRP by Continuous Feeding of Activators: Limitingthe End-Group Loss in the Polymerizations of MethylMethacrylate and Styrene. Polymer 2019, under revision.

\section{ACKNOWLEDGEMENT:}

Financial support by the Board of Regents of the State of Louisiana (LEOSF(2017-20)-RD-A-23) is gratefully acknowledged. 